\title{
Specific Dietary (Poly)phenols Are Associated with Sleep Quality in a Cohort of Italian Adults
}

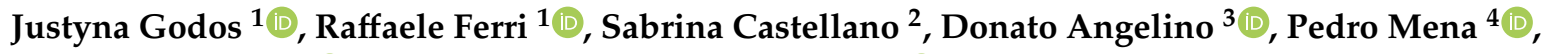 \\ Daniele Del Rio ${ }^{5,6}{ }^{(\mathbb{D}}$, Filippo Caraci ${ }^{1,7}$, Fabio Galvano ${ }^{8}\left(\mathbb{D}\right.$ and Giuseppe Grosso ${ }^{8, *}$ \\ 1 Oasi Research Institute-IRCCS, 94018 Troina, Italy; justyna.godos@gmail.com (J.G.); rferri@oasi.en.it (R.F.); \\ carafil@hotmail.com (F.C.) \\ 2 Department of Educational Sciences, University of Catania, 95124 Catania, Italy; sabrina.castellano@unict.it \\ 3 Faculty of Bioscience and Technology for Food, Agriculture and Environment, University of Teramo, \\ 64100 Teramo, Italy; dangelino@unite.it \\ 4 Human Nutrition Unit, Department of Food and Drugs, University of Parma, 43125 Parma, Italy; \\ pedromiguel.menaparreno@unipr.it \\ 5 School of Advanced Studies on Food and Nutrition, University of Parma, 43125 Parma, Italy; \\ daniele.delrio@unipr.it \\ 6 Department of Veterinary Medicine, University of Parma, 43125 Parma, Italy \\ 7 Department of Drug Sciences, University of Catania, 95125 Catania, Italy \\ 8 Department of Biomedical and Biotechnological Sciences, University of Catania, 95123 Catania, Italy; \\ fgalvano@unict.it \\ * Correspondence: giuseppe.grosso@unict.it; Tel.: +39-095-478-1187
}

Received: 28 March 2020; Accepted: 24 April 2020; Published: 26 April 2020

\begin{abstract}
Background: Diet has been the major focus of attention as a leading risk factor for non-communicable diseases, including mental health disorders. A large body of literature supports the hypothesis that there is a bidirectional association between sleep and diet quality, possibly via the modulation of neuro-inflammation, adult neurogenesis and synaptic and neuronal plasticity. In the present study, the association between dietary total, subclasses of and individual (poly)phenols and sleep quality was explored in a cohort of Italian adults. Methods: The demographic and dietary characteristics of 1936 adults living in southern Italy were analyzed. Food frequency questionnaires (FFQs) were used to assess dietary intake. Data on the (poly)phenol content in foods were retrieved from the Phenol-Explorer database. The Pittsburg Sleep Quality Index was used to measure sleep quality. Multivariate logistic regression analyses were used to test the associations. Results: A significant inverse association between a higher dietary intake of lignans and inadequate sleep quality was found. Additionally, individuals with the highest quartile of hydroxycinnamic acid intake were less likely to have inadequate sleep quality. When individual compounds were taken into consideration, an association with sleep quality was observed for naringenin and apigenin among flavonoids, and for matairesinol among lignans. A secondary analysis was conducted, stratifying the population into normal weight and overweight/obese individuals. The findings in normal weight individuals showed a stronger association between certain classes of, subclasses of and individual compounds and sleep quality. Notably, nearly all individual compounds belonging to the lignan class were inversely associated with inadequate sleep quality. In the overweight/obese individuals, there were no associations between any dietary (poly)phenol class and sleep quality. Conclusions: The results of this study suggest that a higher dietary intake of certain (poly)phenols may be associated with better sleep quality among adult individuals.
\end{abstract}

Keywords: polyphenol; sleep; mental health; cohort; antioxidant; cognitive; brain; Sicily; population 


\section{Introduction}

Diet has been the focus of major attention as a leading risk factor for non-communicable diseases [1,2]. These estimates are based on convincing evidence that dietary factors may play a role in the risks of cardiovascular diseases and certain cancers [3-8]. A more intriguing hypothesis recently explored is that diet may also influence brain health and mental disorders $[9,10]$. A large body of literature supports the hypothesis that there is an association between sleeping patterns and diet quality, possibly mediating weight status and obesity-related disorders [11]. Generally, most of the evidence relies on the positive association between sleep quality or duration and diet quality, but relatively recent studies suggest that a bidirectional relationship may exist, with dietary factors influencing sleep features [12]. Several mechanisms have been hypothesized to explain this association, including inflammation, oxidative stress, the gut microbiome, epigenetic modifications and the direct effects of nutrients and non-nutrients on neuroplasticity [13]. Among the healthy dietary patterns suggested for their putative influence on sleep quality, plant-based foods, including vegetables, grains, nuts, seeds, legumes and fruits, have demonstrated to have a mechanistic relationship with better mental health, potentially influencing sleep features [12]. Those foods are rich sources of bioactive compounds that, in the context of a healthy lifestyle, may play a potential role in preventing subclinical low-grade inflammation, a starting point for several chronic non-communicable diseases as well as for impaired sleep quality and duration $[14,15]$. There is, in fact, evidence that inflammation may mediate a variety of brain disorders involving sleep quality but also stress, depression, dementia and Alzheimer's disease [16]. Thus, it is crucial to understand whether diet may affect the level of inflammation and which compounds should be of major interest.

Dietary (poly)phenols represent a group of compounds present in plant-derived foods that, based on their biochemical structure, may play a pivotal role in radical scavenging and in mediating inflammation processes [17-19]. Several families are commonly consumed when adhering to healthy dietary patterns, including flavonoids (mostly contained in fruits, vegetables, tea and cocoa products), phenolic acids (contained in fruits, coffee, pulses and nuts), stilbenes (mainly contained in wine) and phytoestrogens (including isoflavones and lignans, contained in soy products and legumes). Dietary (poly)phenols have been related to several potential health benefits [20]: recently, they have been hypothesized to also play a role in brain health [21,22]. We previously reported that individuals more adherent to healthy dietary patterns (i.e., the Mediterranean diet) and to a diet with low inflammatory potential were more likely to have higher sleep quality $[23,24]$. In the present study, we aimed to test whether total, subclasses of and individual (poly)phenols may be candidate molecules associated with sleep quality in a cohort of Italian adults.

\section{Materials and Methods}

\subsection{Study Population}

The MEAL study is an observational study aiming to investigate the association between the nutritional and lifestyle habits characterizing the classical Mediterranean area and non-communicable diseases. The baseline data included a sample of 2044 men and women aged 18 or more years old. Individuals were randomly selected in the main districts of the city of Catania, Sicily, Italy. The enrolment and data collection were performed between 2014 and 2015. Details of the study protocol are published elsewhere [25]. All participants were informed about the aims of the study and provided written informed consent. All the study procedures were carried out in accordance with the Declaration of Helsinki (1989) of the World Medical Association. The study protocol has been reviewed and approved by the concerning ethical committee of the Municipal Health Authority (protocol number: 802/23 December 2014). 


\subsection{Data Collection}

Electronic data collection was performed by face-to-face assisted personal interviews, using tablet computers. In order to visualize the response options, participants were provided with a paper copy of the questionnaire. However, final answers were registered directly by the interviewer. The demographic data included gender, age at recruitment, highest educational degree achieved, occupation (specifying the nature of the most important employment during the year before the investigation) or last occupation before retirement, and marital status. Educational status was categorized as (i) low (primary/secondary), (ii) medium (high school), and (iii) high (university). Occupational status was categorized as (i) unemployed, (ii) low (unskilled workers), (iii) medium (partially skilled workers), and (iv) high (skilled workers). Physical activity status was evaluated using International Physical Activity Questionnaires (IPAQ) [26], which demonstrated an acceptable validity for the Italian population (the Cronbach's alpha values were 0.73 and 0.60 for the short and long versions, respectively) [27]: the instrument included a set of questionnaires (five domains) investigating the time spent being physically active in the last 7 days. Based on the IPAQ guidelines, the final score allows categorizing physical activity levels as (i) low, (ii) moderate, and (iii) high. Smoking status was categorized as (i) non-smoker, (ii) ex-smoker, and (iii) current smoker. Alcohol consumption was categorized as (i) none, (ii) moderate drinker (0.1-12 g/d) and (iii) regular drinker $(>12 \mathrm{~g} / \mathrm{d})$. Anthropometric measurements have been collected following standard procedures [28]. Arterial blood pressure was measured in sitting position and after at least 5 min of rest, at the end of the physical examination. Because of the possibility of differences in blood pressure measurements, the measurements were taken three times at the right arm, relaxed and well supported by a table, with an angle of $45^{\circ}$ from the trunk. A mean of the last two measurements was considered for inclusion in the database. Patients were considered hypertensive when their average systolic/diastolic blood pressure levels were higher than or equal to $140 / 90 \mathrm{~mm} \mathrm{Hg}$, they were taking anti-hypertensive medications, or they had previously been diagnosed with hypertension.

\subsection{Dietary Assessment}

The dietary assessment was performed by the administration of two food frequency questionnaires (FFQ, long and short versions) that had been previously tested for validity and reliability for individuals living in Sicily $[29,30]$. For the purposes of this study, the data from the most comprehensive FFQ including 110 food items were used. The identification of food intake, energy content and macro- and micro-nutrient intake was performed through comparison with the food composition tables from the Research Center for Foods and Nutrition [31]. The intake of seasonal foods referred to consumption during the period in which the food was available, proportionally adjusted by its intake in one year. The instrument showed a good relative validity (all major food groups with the exception of bread and soft drinks had significant Person's correlation coefficients, over 0.60, and the highest correlation coefficients for coffee ( $R=0.96$ in men and women), tea $(R=0.79$ in men and 0.80 in women) and alcoholic beverages ( $R=0.83$ in men and 0.88 in women)) and reliability (all major food groups besides bread had significant Person's correlation coefficients, over 0.60 , and the highest correlation coefficients for coffee ( $R=0.97$ in men and $R=0.96$ in women), tea $(R=0.82$ in men and 0.84 in women) and alcoholic beverages $(R=0.87$ in men and 0.92 in women $)$ ). FFQs with unreliable intakes $(<1000$ or $>6000 \mathrm{kcal} / \mathrm{d}$ ) were excluded from the analyses $(n=107)$, leaving a total of 1936 individuals included in the analysis.

\subsection{Estimation of Polyphenol Intake}

The process of the estimation of habitual (poly)phenol intakes has been previously described in detail [32]. Briefly, data on the (poly)phenol content in foods were retrieved from the Phenol-Explorer database (www.phenol-explorer.eu) [33]. A new version of the Phenol-Explorer database containing data on the effects of cooking and food processing on (poly)phenol contents was used whenever possible, 
in order to apply (poly)phenol-specific retention factors [34]. Foods that contained no (poly)phenols were excluded from the calculation, leaving a total of 75 items included in the analyses. Food weight loss or gain during cooking was corrected using yield factors [35]. Average food consumption was calculated (in $\mathrm{g}$ or $\mathrm{mL}$ ) by following the standard portion sizes used in the study and then converted to $24 \mathrm{~h}$ intake. Finally, a search was carried out in the Phenol-Explorer database to retrieve the mean content values for all (poly)phenols contained in the selected foods. Next, (poly)phenol intake from each food was calculated by multiplying the content of each (poly)phenol class by the daily consumption of each food. The total (poly)phenol intake was considered as the sum of all the main classes and subclasses. Finally, (poly)phenol intake was adjusted for total energy intake (kcal/d) using the residual method [36].

\subsection{Sleep Quality}

The Pittsburg sleep quality index (PSQI) [37] was used to assess participants' sleep quality and disturbances in the past six months, which has also been demonstrated to be a good and reliable tool in the Italian population (the internal consistency was represented by a Cronbach's alpha of 0.835) [38]. It consists of 19 items that are rated on a four-point scale (0-3) and grouped into seven components (sleep quality, sleep latency, sleep duration, habitual sleep efficiency, sleep disturbance, the use of sleeping medications, and daytime dysfunction). The item scores in each component were summed and converted to component scores ranging from 0 (better) to 3 (worse) based on guidelines. The total PSQI score was calculated as the summation of seven component scores, ranging from 0 to 21 , where a higher score indicates a worse condition. A score of $<5$ on for total global PSQI is indicative of adequate sleep quality.

\subsection{Statistical Analysis}

Frequencies are expressed as absolute numbers and percentages; continuous variables are expressed as means and standard deviations. Individuals were divided into quartiles of dietary (poly)phenol intake and the distributions of background characteristics were compared between the groups. Differences were tested with Chi-square tests for categorical variables, ANOVA for continuous variables distributed normally, and Kruskall-Wallis tests for variables not normally distributed. Energy-adjusted multivariate logistic regression models were used to test the association between the variables of exposure (including total (poly)phenols, their classes, subclasses and individual compounds) and inadequate sleep quality; a multivariate model adjusted for all other background characteristics (body mass index, physical activity, educational status, occupational status, smoking status, alcohol consumption, occurrence of hypertension, diabetes, dyslipidemias, cardiovascular disease, cancer, and menopausal status) was also used to test whether the observed associations were independent from the aforementioned variables. All reported $P$-values were based on two-sided tests and compared to a significance level of $5 \%$. Bonferroni correction was applied and $P$-values meeting the threshold of 0.05 divided by the number of polyphenol quartiles were noted. The SPSS 17 (SPSS Inc., Chicago, IL, USA) software was used for all the statistical calculations.

\section{Results}

The baseline characteristics of the study population by quartiles of energy-adjusted total (poly)phenol intake are presented in Table 1. The distributions of certain variables, such as age and education level, did not follow linear trends, as individuals in the middle quartiles were significantly older and had lower educational levels than the others. The participants in the highest quartile of total (poly)phenol intake had moderate levels of physical activity and were moderate or regular alcohol drinkers, and they also had a lower prevalence of hypertension, while concerning type 2 diabetes, the distribution was not linear and higher rates were registered in the middle quartiles (Table 1). High total (poly)phenol intake was also correlated with higher total energy intake (Table 1). 
Table 1. Background characteristics of participants in the MEAL cohort by quartiles of total (poly)phenol intake (energy-adjusted).

\begin{tabular}{|c|c|c|c|c|c|}
\hline & \multicolumn{4}{|c|}{ Total (Poly)phenol Intake } & \multirow{2}{*}{$P$} \\
\hline & Q1 & Q2 & Q3 & Q4 & \\
\hline Age (years), mean (SD) & $47.0(19.3)$ & $48.9(18.0)$ & $50.1(16.7)$ & $47.6(16.4)$ & 0.036 \\
\hline Men, $n(\%)$ & $193(43.0)$ & $217(43.4)$ & $195(39.0)$ & 199 (40.9) & 0.472 \\
\hline BMI, mean (SD) & $25.9(4.4)$ & $25.9(4.8)$ & $25.9(4.5)$ & $25.5(4.6)$ & 0.393 \\
\hline Smoking status, $n(\%)$ & & & & & 0.598 \\
\hline Current & $96(21.4)$ & $127(25.4)$ & $130(26.0)$ & $112(23.0)$ & \\
\hline Former & $62(13.8)$ & $71(14.2)$ & $75(15.0)$ & $68(14.0)$ & \\
\hline Never & $291(64.8)$ & $302(60.4)$ & $295(59.0)$ & $307(63.0)$ & \\
\hline Educational level, $n(\%)$ & & & & & 0.001 \\
\hline Low & $147(32.7)$ & $185(37.0)$ & $187(37.4)$ & $178(36.6)$ & \\
\hline Medium & $153(34.1)$ & $180(36.0)$ & $213(42.6)$ & $174(35.7)$ & \\
\hline High & $149(33.2)$ & $135(27.0)$ & $100(20.0)$ & $135(27.7)$ & \\
\hline Occupational level, $n(\%)$ & & & & & 0.046 \\
\hline Unemployed & $90(23.7)$ & $115(26.7)$ & $131(28.8)$ & 125 (31.9) & \\
\hline Low & $64(16.8)$ & $66(15.3)$ & $74(16.3)$ & $62(15.8)$ & \\
\hline Medium & $87(22.9)$ & $126(29.2)$ & $123(27.0)$ & $104(26.5)$ & \\
\hline High & $139(36.6)$ & $124(28.8)$ & $127(27.9)$ & $101(25.8)$ & \\
\hline Physical activity level, $n(\%)$ & & & & & 0.010 \\
\hline Low & $82(20.4)$ & $92(20.4)$ & $69(19.7)$ & $86(19.7)$ & \\
\hline Medium & $192(47.8)$ & $236(52.2)$ & $200(45.8)$ & $228(52.2)$ & \\
\hline High & $128(31.8)$ & $124(27.4)$ & $168(38.4)$ & $123(28.1)$ & \\
\hline Alcohol consumption, $n(\%)$ & & & & & $<0.001$ \\
\hline None & $125(27.8)$ & $118(23.6)$ & $74(14.8)$ & $58(11.9)$ & \\
\hline Moderate $(0.1-12 \mathrm{~g} / \mathrm{d})$ & $317(70.6)$ & $340(67.9)$ & $306(61.2)$ & $243(49.9)$ & \\
\hline $\operatorname{Regular}(>12 \mathrm{~g} / \mathrm{d})$ & $7(1.6)$ & $43(8.6)$ & $120(24.0)$ & $186(38.2)$ & \\
\hline \multicolumn{6}{|l|}{ Health status, $n(\%)$} \\
\hline Hypertension & $240(53.5)$ & $275(54.9)$ & $261(52.2)$ & $200(41.1)$ & $<0.001$ \\
\hline Diabetes & $21(4.7)$ & $50(10.0)$ & $41(8.2)$ & $34(7.0)$ & 0.018 \\
\hline Dyslipidemias & $69(15.4)$ & $100(20.0)$ & $102(20.4)$ & $85(17.5)$ & 0.158 \\
\hline Cardiovascular disease & $40(9.1)$ & $36(7.4)$ & $42(8.7)$ & $36(7.6)$ & 0.732 \\
\hline Cancer & $17(3.8)$ & $18(3.6)$ & $18(3.6)$ & $25(5.1)$ & 0.556 \\
\hline Menopausal status (women only), $n(\%)$ & $118(45.4)$ & $129(44.6)$ & $146(46.9)$ & $133(44.5)$ & 0.926 \\
\hline Total energy intake (kcal/d), mean (SD) & $\begin{array}{l}1749.9 \\
(563.4)\end{array}$ & $\begin{array}{l}1916.2 \\
(552.7)\end{array}$ & $\begin{array}{l}2062.8 \\
(625.2)\end{array}$ & $\begin{array}{c}2704.2 \\
(1068.9)\end{array}$ & $<0.001$ \\
\hline
\end{tabular}

A total of 509 individuals (32.4\%) reported inadequate sleep quality. No association between total or individual major classes of (poly)phenols and sleep quality was found, with the exception of lignans, for which participants in the third quartile of intake were less likely to have inadequate sleep quality after adjusting for potential confounding factors (OR $=0.62 ; 95 \%$ CI: $0.43,0.88$; Table 2). None of the flavonoid subclasses showed an association with sleep quality. Conversely, individuals with the highest quartile of hydroxycinnamic acid intake were less likely to have inadequate sleep quality ( $\mathrm{OR}=0.67 ; 95 \%$ CI: 0.46, 0.98; Table 2). When individual compounds were taken into consideration, inverse associations with inadequate sleep quality were observed for naringenin (OR $=0.66 ; 95 \%$ CI: $0.46,0.95)$ and apigenin (OR $=0.63 ; 95 \%$ CI: 0.44, 0.90) among flavonoids, and for matairesinol $(\mathrm{OR}=0.66 ; 95 \%$ CI: 0.46, 0.96) among lignans (Table 2). 
Table 2. Odds ratios (ORs) and 95\% confidence intervals (CIs) for the associations between (poly)phenol intake (total, main classes, subclasses and individual compounds) and adequate sleep quality in the MEAL cohort.

\begin{tabular}{|c|c|c|c|c|c|}
\hline & \multicolumn{4}{|c|}{ (Poly)phenol Quartiles, OR (95\% CI) } & \multirow[b]{2}{*}{$P$ for Trend } \\
\hline & Q1 & Q2 & Q3 & Q4 & \\
\hline Total (poly)phenols & 1 & $1.12(0.80,1.58)$ & $0.87(0.61,1.25)$ & $1.04(0.69,1.55)$ & 0.476 \\
\hline Total flavonoids & 1 & $0.83(0.58,1.18)$ & $0.92(0.64,1.31)$ & $0.97(0.66,1.43)$ & 0.948 \\
\hline Flavanols & 1 & $0.90(0.63,1.28)$ & $0.93(0.66,1.32)$ & $1.25(0.89,1.77)$ & 0.603 \\
\hline Catechins & 1 & $0.81(0.57,1.15)$ & $0.96(0.67,1.38)$ & $1.05(0.73,1.50)$ & 0.255 \\
\hline Flavonols & 1 & $1.07(0.68,1.67)$ & $0.90(0.48,1.68)$ & $1.32(0.59,2.92)$ & 0.645 \\
\hline Quercetin & 1 & $1.44(0.97,2.11)$ & $1.51(1.04,2.20)$ & $1.33(0.88,2.01)$ & 0.368 \\
\hline Kaempferol & 1 & $0.72(0.50,1.02)$ & $0.74(0.53,1.05)$ & $0.91(0.59,1.41)$ & 0.104 \\
\hline Flavanones & 1 & $1.03(0.72,1.45)$ & $0.75(0.53,1.06)$ & $0.72(0.50,1.03)$ & 0.263 \\
\hline Hesperetin & 1 & $0.85(0.60,1.21)$ & $0.96(0.68,1.36)$ & $0.81(0.58,1.15)$ & 0.249 \\
\hline Naringenin & 1 & $0.76(0.54,1.08)$ & $0.62(0.44,0.89)$ & $0.66(0.46,0.95)$ & 0.020 \\
\hline Flavones & 1 & $1.03(0.72,1.45)$ & $0.75(0.53,1.06)$ & $0.72(0.50,1.03)$ & 0.016 \\
\hline Apigenin & 1 & $0.67(0.46,0.96)$ & $0.61(0.41,0.90)$ & $0.63(0.44,0.90)$ & 0.046 \\
\hline Luteolin & 1 & $1.09(0.77,1.53)$ & $0.85(0.60,1.20)$ & $0.84(0.56,1.15)$ & 0.043 \\
\hline Anthocyanins & 1 & $0.91(0.63,1.31)$ & $0.97(0.68,1.37)$ & $0.86(0.59,1.26)$ & 0.459 \\
\hline Isoflavones & 1 & $0.90(0.64,1.27)$ & $0.95(0.68,1.33)$ & $0.92(0.65,1.30)$ & 0.356 \\
\hline Daidzein & 1 & $0.90(0.64,1.27)$ & $0.98(0.70,1.38)$ & $0.89(0.62,1.27)$ & 0.301 \\
\hline Genistein & 1 & $0.90(0.64,1.27)$ & $0.93(0.66,1.32)$ & $0.93(0.65,1.32)$ & 0.263 \\
\hline Biochanin A & 1 & $0.75(0.51,1.10)$ & $0.96(0.66,1.41)$ & $1.07(0.72,1.60)$ & 0.025 \\
\hline Phenolic acids & 1 & $1.32(0.93,1.87)$ & $1.31(0.97,1.87)$ & $1.16(0.81,1.65)$ & 0.457 \\
\hline Hydroxybenzoic acids & 1 & $1.10(0.80,1.53)$ & $1.07(0.74,1.53)$ & $1.13(0.79,1.61)$ & 0.852 \\
\hline Vanillic acid & 1 & $1.00(0.69,1.44)$ & $1.34(0.93,1.93)$ & $1.27(0.85,1.89)$ & 0.624 \\
\hline Hydroxycinnamic acids & 1 & $0.89(0.63,1.26)$ & $0.77(0.51,1.09)$ & $0.67(0.46,0.98)$ & $0.005^{\mathrm{a}}$ \\
\hline Caffeic acid & 1 & $0.95(0.67,1.36)$ & $0.72(0.50,1.04)$ & $0.88(0.54,1.43)$ & 0.055 \\
\hline Cinnamic acid & 1 & $1.02(0.73,1.42)$ & $0.93(0.65,1.35)$ & $1.09(0.77,1.52)$ & 0.680 \\
\hline Ferulic acid & 1 & $0.71(0.50,1.01)$ & $0.94(0.67,1.31)$ & $0.77(0.53,1.12)$ & 0.299 \\
\hline Stilbenes & 1 & $0.73(0.50,1.06)$ & $0.83(0.56,1.24)$ & $0.97(0.60,1.56)$ & 0.828 \\
\hline Lignans & 1 & $0.85(0.60,1.21)$ & $0.62(0.43,0.88)$ & $0.78(0.54,1.12)$ & 0.051 \\
\hline Lariciresinol & 1 & $0.90(0.64,1.27)$ & $0.69(0.49,0.98)$ & $0.85(0.59,1.22)$ & 0.187 \\
\hline Matairesinol & 1 & $0.72(0.51,1.02)$ & $0.67(0.47,0.95)$ & $0.66(0.46,0.96)$ & 0.017 \\
\hline Pinoresinol & 1 & $0.90(0.64,1.27)$ & $0.64(0.45,0.92)$ & $0.78(0.54,1.12)$ & 0.090 \\
\hline Secoisolariciresinol & 1 & $0.72(0.51,1.02)$ & $0.68(0.48,0.98)$ & $0.80(0.55,1.16)$ & 0.187 \\
\hline
\end{tabular}

Adjusted for total energy intake (continuous), body mass index (continuous), physical activity (low/medium/high), educational status (low/medium/high), occupational status (unemployed/low/medium/high), smoking status (current/former/never), alcohol consumption (none/moderate/regular), occurrence of hypertension, diabetes, dyslipidemias, cardiovascular disease, cancer (yes/no), and menopausal status (women only, yes/no). ${ }^{\text {a }} P$-value meeting threshold for Bonferroni correction.

A secondary analysis was conducted, stratifying the population into normal weight and overweight/obese individuals. The findings in normal weight individuals showed a stronger association between certain classes, subclasses and individual compounds and sleep quality (Table 3). Notably, nearly all individual compounds belonging to the lignan class (secoisolariciresinol, matairesinol and pinoresinol) were inversely associated with inadequate sleep quality (Table 3). 
Table 3. Odds ratios (ORs) and 95\% confidence intervals (CIs) for the associations between (poly)phenol intake (total, main classes, subclasses and individual compounds) and adequate sleep quality in normal weight individuals.

\begin{tabular}{|c|c|c|c|c|c|}
\hline & \multicolumn{4}{|c|}{ (Poly)phenol Quartiles, OR (95\% CI) } & \multirow[b]{2}{*}{$P$ for Trend } \\
\hline & Q1 & Q2 & Q3 & Q4 & \\
\hline Total (poly)phenols & 1 & $0.74(0.45,1.21)$ & $0.52(0.31,0.89)$ & $0.70(0.39,1.25)$ & 0.060 \\
\hline Total flavonoids & 1 & $0.60(0.36,1.01)$ & $0.60(0.36,1.01)$ & $0.66(0.38,1.13)$ & 0.308 \\
\hline Flavanols & 1 & $0.88(0.44,1.75)$ & $0.66(0.25,1.71)$ & $1.21(0.39,3.75)$ & 0.571 \\
\hline Catechins & 1 & $0.76(0.45,1.27)$ & $0.84(0.49,1.42)$ & $0.94(0.57,1.55)$ & 0.869 \\
\hline Flavonols & 1 & $0.86(0.52,1.45)$ & $0.78(0.47,1.29)$ & $1.03(0.62,1.71)$ & 0.914 \\
\hline Quercetin & 1 & $1.24(0.71,2.16)$ & $1.55(0.90,2.68)$ & $1.17(0.63,2.16)$ & 0.827 \\
\hline Kaempferol & 1 & $0.65(0.39,1.09)$ & $0.68(0.42,1.10)$ & $1.00(0.53,1.87)$ & 0.228 \\
\hline Flavanones & 1 & $1.32(0.78,2.23)$ & $1.05(0.61,1.81)$ & $0.88(0.50,1.52)$ & 0.774 \\
\hline Hesperetin & 1 & $1.24(0.74,2.09)$ & $0.96(0.56,1.65)$ & $0.87(0.50,1.50)$ & 0.724 \\
\hline Naringenin & 1 & $0.80(0.48,1.32)$ & $0.51(0.30,0.85)$ & $0.49(0.28,0.85)$ & 0.016 \\
\hline Flavones & 1 & $0.94(0.56,1.56)$ & $0.64(0.38,1.07)$ & $0.52(0.30,0.91)$ & 0.037 \\
\hline Apigenin & 1 & $0.78(0.45,1.34)$ & $0.61(0.34,1.07)$ & $0.67(0.40,1.13)$ & 0.062 \\
\hline Luteolin & 1 & $1.34(0.82,2.17)$ & $0.77(0.47,1.28)$ & $0.68(0.39,1.18)$ & 0.106 \\
\hline Anthocyanins & 1 & $1.04(0.63,1.69)$ & $0.73(0.44,1.21)$ & $0.59(0.34,1.03)$ & 0.033 \\
\hline Isoflavones & 1 & $0.99(0.59,1.63)$ & $1.11(0.66,1.84)$ & $1.00(0.61,1.63)$ & 0.878 \\
\hline Daidzein & 1 & $1.04(0.63,1.72)$ & $1.14(0.68,1.91)$ & $1.02(0.62,1.68)$ & 0.803 \\
\hline Genistein & 1 & $1.06(0.64,1.75)$ & $1.02(0.59,1.76)$ & $1.09(0.66,1.78)$ & 0.992 \\
\hline Biochanin A & 1 & $0.59(0.33,1.04)$ & $0.79(0.46,1.37)$ & $0.89(0.49,1.60)$ & 0.823 \\
\hline Phenolic acids & 1 & $1.22(0.72,2.05)$ & $1.18(0.70,1.96)$ & $0.79(0.46,1.35)$ & 0.538 \\
\hline Hydroxybenzoic acids & 1 & $1.24(0.76,2.00)$ & $0.73(0.42,1.27)$ & $1.15(0.67,1.97)$ & 0.631 \\
\hline Vanillic acid & 1 & $0.93(0.55,1.56)$ & $1.18(0.71,1.97)$ & $1.31(0.74,2.31)$ & 0.816 \\
\hline Hydroxycinnamic acids & 1 & $0.59(0.36,0.97)$ & $0.60(0.37,0.97)$ & $0.39(0.22,0.69)$ & 0.049 \\
\hline Caffeic acid & 1 & $1.22(0.74,1.99)$ & $0.49(0.29,0.84)$ & $0.86(0.42,1.79)$ & 0.044 \\
\hline Cinnamic acid & 1 & $0.84(0.52,1.35)$ & $0.88(0.52,1.49)$ & $0.73(0.44,1.21)$ & 0.481 \\
\hline Ferulic acid & 1 & $0.87(0.51,1.47)$ & $0.92(0.55,1.54)$ & $0.91(0.52,1.60)$ & 0.312 \\
\hline Stilbenes & 1 & $0.57(0.33,0.99)$ & $0.79(0.45,1.39)$ & $0.95(0.43,2.09)$ & 0.553 \\
\hline Lignans & 1 & $0.70(0.42,1.15)$ & $0.45(0.27,0.77)$ & $0.54(0.31,0.94)$ & 0.040 \\
\hline Lariciresinol & 1 & $0.79(0.48,1.30)$ & $0.54(0.32,0.90)$ & $0.61(0.36,1.05)$ & 0.061 \\
\hline Matairesinol & 1 & $0.70(0.42,1.16)$ & $0.57(0.35,0.95)$ & $0.50(0.29,0.87)$ & 0.025 \\
\hline Pinoresinol & 1 & $0.68(0.41,1.12)$ & $0.47(0.27,0.80)$ & $0.55(0.32,0.96)$ & 0.030 \\
\hline Secoisolariciresinol & 1 & $0.54(0.33,0.88)$ & $0.54(0.31,0.92)$ & $0.51(0.29,0.89)$ & 0.047 \\
\hline
\end{tabular}

Adjusted for total energy intake (continuous), body mass index (continuous), physical activity (low/medium/high), educational status (low/medium/high), occupational status (unemployed/low/medium/high), smoking status (current/former/never), alcohol consumption (none/moderate/regular), occurrence of hypertension, diabetes, dyslipidemias, cardiovascular disease, cancer (yes/no), and menopausal status (women only, yes/no).

To the contrary, in the overweight/obese individuals, there were no associations between any dietary (poly)phenol class and sleep quality. Among the individual components, only apigenin was significantly associated with sleep quality (OR $=0.53 ; 95 \%$ CI: 0.31, 0.90) (Table 4). 
Table 4. Odds ratios (ORs) and 95\% confidence intervals (CIs) for the associations between (poly)phenol intake (total, main classes, subclasses and individual compounds) and adequate sleep quality in overweight/obese individuals.

\begin{tabular}{|c|c|c|c|c|c|}
\hline & \multicolumn{4}{|c|}{ (Poly)phenol Quartiles, OR (95\% CI) } & \multirow[b]{2}{*}{$P$ for Trend } \\
\hline & Q1 & Q2 & Q3 & Q4 & \\
\hline Total (poly)phenols & 1 & $1.64(0.98,2.73)$ & $1.13(0.82,2.29)$ & $1.57(0.86,2.83)$ & 0.219 \\
\hline Total flavonoids & 1 & $1.24(0.73,2.10)$ & $1.50(0.88,2.55)$ & $1.69(0.93,3.05)$ & 0.075 \\
\hline Flavanols & 1 & $1.37(0.73,2.56)$ & $1.10(0.46,2.60)$ & $1.37(0.42,4.46)$ & 0.982 \\
\hline Catechins & 1 & $0.88(0.54,1.43)$ & $1.26(0.74,2.12)$ & $1.31(0.77,2.24)$ & 0.114 \\
\hline Flavonols & 1 & $0.89(0.53,1.48)$ & $1.16(0.71,1.91)$ & $1.47(0.90,2.40)$ & 0.183 \\
\hline Quercetin & 1 & $1.55(0.88,2.73)$ & $1.44(0.83,2.49)$ & $1.46(0.82,2.60)$ & 0.180 \\
\hline Kaempferol & 1 & $0.90(0.53,1.53)$ & $0.97(0.57,1.63)$ & $0.93(0.49,1.76)$ & 0.918 \\
\hline Flavanones & 1 & $0.61(0.37,1.01)$ & $1.00(0.62,1.61)$ & $0.82(0.51,1.32)$ & 0.847 \\
\hline Hesperetin & 1 & $0.60(0.36,1.00)$ & $0.99(0.62,1.59)$ & $0.82(0.51,1.33)$ & 0.803 \\
\hline Naringenin & 1 & $0.86(0.51,1.43)$ & $0.77(0.46,1.28)$ & $0.99(0.59,1.65)$ & 0.899 \\
\hline Flavones & 1 & $1.00(0.61,1.64)$ & $0.81(0.50,1.33)$ & $0.89(0.54,1.47)$ & 0.195 \\
\hline Apigenin & 1 & $0.56(0.33,0.95)$ & $0.61(0.34,1.07)$ & $0.53(0.31,0.90)$ & 0.555 \\
\hline Luteolin & 1 & $0.80(0.47,1.34)$ & $0.79(0.48,1.30)$ & $0.88(0.53,1.45)$ & 0.208 \\
\hline Anthocyanins & 1 & $0.84(0.48,1.48)$ & $1.17(0.69,1.97)$ & $1.29(0.73,2.27)$ & 0.155 \\
\hline Isoflavones & 1 & $0.87(0.53,1.41)$ & $0.96(0.60,1.53)$ & $0.91(0.54,1.54)$ & 0.349 \\
\hline Daidzein & 1 & $0.81(0.50,1.32)$ & $0.97(0.60,1.55)$ & $0.84(0.49,1.44)$ & 0.269 \\
\hline Genistein & 1 & $0.80(0.49,1.30)$ & $0.94(0.58,1.52)$ & $0.85(0.50,1.43)$ & 0.286 \\
\hline Biochanin A & 1 & $1.02(0.58,1.79)$ & $1.27(0.73,2.23)$ & $1.45(0.81,2.60)$ & 0.024 \\
\hline Phenolic acids & 1 & $1.35(0.83,2.20)$ & $1.38(0.83,2.28)$ & $1.49(0.90,2.45)$ & 0.079 \\
\hline Hydroxybenzoic acids & 1 & $0.94(0.58,1.51)$ & $1.41(0.85,2.35)$ & $1.06(0.64,1.76)$ & 0.308 \\
\hline Vanillic acid & 1 & $1.04(0.60,1.80)$ & $1.39(0.80,2.39)$ & $1.14(0.63,2.04)$ & 0.324 \\
\hline Hydroxycinnamic acids & 1 & $1.28(0.77,2.12)$ & $0.86(0.50,1.47)$ & $1.03(0.60,1.78)$ & 0.260 \\
\hline Caffeic acid & 1 & $0.69(0.40,1.20)$ & $0.90(0.52,1.52)$ & $0.81(0.40,1.62)$ & 0.860 \\
\hline Cinnamic acid & 1 & $1.22(0.75,2.00)$ & $0.99(0.58,1.69)$ & $1.53(0.93,2.51)$ & 0.195 \\
\hline Ferulic acid & 1 & $0.61(0.37,1.00)$ & $0.92(0.58,1.47)$ & $0.72(0.42,1.22)$ & 0.609 \\
\hline Stilbenes & 1 & $0.99(0.57,1.69)$ & $0.84(0.46,1.53)$ & $0.91(0.48,1.72)$ & 0.555 \\
\hline Lignans & 1 & $1.15(0.69,1.91)$ & $0.86(0.52,1.43)$ & $1.23(0.73,2.07)$ & 0.538 \\
\hline Lariciresinol & 1 & $1.09(0.66,1.80)$ & $0.90(0.55,1.47)$ & $1.30(0.77,2.17)$ & 0.396 \\
\hline Matairesinol & 1 & $0.86(0.52,1.44)$ & $0.84(0.50,1.41)$ & $0.99(0.58,1.69)$ & 0.864 \\
\hline Pinoresinol & 1 & $1.13(0.69,1.84)$ & $0.84(0.50,1.41)$ & $1.11(0.67,1.86)$ & 0.520 \\
\hline Secoisolariciresinol & 1 & $1.02(0.62,1.70)$ & $0.90(0.54,1.51)$ & $1.37(0.80,2.33)$ & 0.342 \\
\hline
\end{tabular}

Adjusted for total energy intake (continuous), body mass index (continuous), physical activity (low/medium/high), educational status (low/medium/high), occupational status (unemployed/low/medium/high), smoking status (current/former/never), alcohol consumption (none/moderate/regular), occurrence of hypertension, diabetes, dyslipidemias, cardiovascular disease, cancer (yes/no), and menopausal status (women only, yes/no).

\section{Discussion}

In this article, we tested whether dietary (poly)phenols were associated with sleep quality in a cohort of Italian adults. Individuals showing a higher intake of some flavonoid subclasses (flavanones and flavones), phenolic acids (such as hydroxycinnamic acids) and lignans were significantly less likely to have inadequate sleep quality. These findings suggest that some classes of (poly)phenol may play a specific role when exploring their relationship with brain and mental health. Interestingly, the associations were more evident when stratifying the cohort by weight status, showing significant results in normal weight individuals, but no confirmed associations for overweight/obese participants.

To date, only one recent study has investigated the relationship between dietary (poly)phenols derived from fruit and vegetables and sleep duration [39]. The study was conducted on 13,958 women with about 4 years of follow-up in the UK Women's Cohort Study: total fruit and vegetable consumption and their estimated content of total polyphenols were directly associated with sleep duration, while individual (poly)phenol classes were not associated with the outcome of interest [39]. Despite no other studies being focused on polyphenols, some studies reported a direct relationship between 
sleep duration and quality, and fruit and vegetable intake [40,41]. Other studies showed the role of certain polyphenol-rich foods (i.e., black tea and cocoa products) in improving sleep quality $[42,43]$. Despite there being no other studies specifically conducted on (poly)phenols and sleep quality, there is consistent evidence from the literature suggesting a potential role of dietary (poly)phenols in improving mental health and preventing conditions that are associated with sleep disorders. For instance, some cohort studies showed that individuals with higher intakes of the same flavonoid, hydroxycinnamic and lignan classes found to be significantly associated with better sleep quality in this study were less likely to have depressive symptoms [44,45]. Other studies also showed an inverse association between fruit and vegetable intake and depressive symptoms and perceived stress, despite most of them have been conducted on students [46-49].

From a general mechanistic point of view, (poly)phenol circulating metabolites are able to pass through the blood-brain barrier to various extents, depending on their degree of lipophilicity, with less polar (poly)phenol metabolites capable of greater brain uptake than more polar ones [50,51]. The main potential beneficial effects of dietary (poly)phenols in the central nervous system include the suppression of neuronal apoptosis, modulation of signaling pathways implicated in neuron survival, and stimulation of adult neurogenesis [52-54]. With special regard to specific mechanisms related to sleep features, dietary (poly)phenols have been shown to improve resilience after sleep deprivation [55]; some individual molecules, such as apigenin, are able to reduce locomotor activity, prolong sleep time, increase sleep rate increase and sleep time in combined administration with a GABA(A) receptor agonist, and show synergic effects in potentiating sleep onset in animal models [56-58]. Additionally, derivates of hydroxycinnamic acids have been identified as agonists for both gamma-amino butyric acid (GABA) receptors and act synergistically with 5-hydroxytryptophan (5-HTP), both of which play a role in sleep quality, including having sedative effects on locomotion activity, prolonging sleeping time and shortening sleep latency [59-61].

Dietary (poly)phenols have been shown to decrease systemic inflammation [62] but also exert anti-neuroinflammatory properties and reduce oxidative stress and inflammation-related conditions [63]. Several studies have shown that molecules of interest from our study, including some flavonoids (i.e., apigenin) and hydroxycinnamic acids, improve cell antioxidant activity against oxidative stress in the central nervous system $[64,65]$. Additionally, lignans have been demonstrated to exert anti-oxidative and anti-inflammatory properties in neurons and protect the blood-brain barrier against inflammatory cells by reducing oxidative stress, inflammation and permeability [66-68]. Dietary (poly)phenols may ameliorate poor endothelial function [69] and help to control blood pressure [70], which, in turn, has been associated with measures of sleep quality together with decreases in the percentage of REM sleep and increases in REM sleep latency [71-73]. Previous epidemiological studies have shown an inverse association between the intake of specific classes of (poly)phenol (in line with the findings shown in the present study) —including flavones and flavanones among flavonoids [74], and hydroxycinnamic acids-and the occurrence of hypertension $[75,76]$. The mechanisms underlying these relationships are still under investigation; besides, regarding the direct effect of (poly)phenols (especially hydroxycinnamic acids) on low-grade inflammation, which, in turn, may affect endothelial function $[77,78]$, an intriguing hypothesis involves nitric oxide-mediated vasodilation in the brain, which has been shown to facilitate REM sleep [79].

An emerging body of literature investigates the double interexchange of information between the gut microbiota and the brain through a complex system of signals involving neural, endocrine and inflammatory mechanisms [80]. In fact, the gut microbiota has been shown to affect brain and behaviors related to anxiety and depressive symptoms depending on bacterial family ratios, dysbiosis, and subsequent modulation through dietary (poly)phenol intake [81]; the status of the pro- and anti-inflammatory balance in the gut has been demonstrated to have an impact at the systemic level and on the central nervous system [82,83]. Recent studies show that dietary (poly)phenols may play a role in the modulation of gut microbiota metabolism and that variations in the gut microbiota can affect (poly)phenol activity [84]. This hypothesis is particularly valid in light of our results stratified 
by weight status; in the intestinal microbiota of obese people, a specific increase in the proportion of class Firmicutes to class Bacteroidetes has been shown compared to in normal-weight individuals [85], which may affect (poly)phenol transformation and absorption in the gut [86] and their anti-oxidant effects [87-89]. However, current evidence is still limited, largely based on cell and animal studies, and future studies conducted on humans are needed to identify specific metabotypes associated with activity in the brain.

To the best of our knowledge, this is the first study investigating such a comprehensive group of compounds in order to identify key (poly)phenol molecules of potential interest to improve sleep quality. Moreover, based on our previous results [32], no unique food source is responsible for specific compounds or classes of (poly)phenols; thus, the present analysis is able to detect the potential role of (poly)phenols rather than of the individual foods underlying their consumption. However, the findings presented in this study should be considered in light of some limitations. Firstly, this study provided evidence from a cross-sectional analysis, which cannot exclude reverse causation nor describe a causal relation. Secondly, all methods used to assess food consumption and dietary polyphenol intake provide only estimations, while true intake cannot be estimated without measuring biomarkers or metabolites. Despite the use of the Phenol-Explorer database being validated and widespread, this method cannot take into account molecular transformation or interaction. Moreover, recall bias and unmeasured confounding factors (i.e., jobs requiring night shifts) should be considered as potential limitations. However, these methods are commonly used in the current scientific literature, representing the standard for scientific research until new methods are validated and made available. Thirdly, no other aspects related to sleeping problems or other mental health issues have been considered, while they may be associated with sleep quality. Thus, the potential mediating effect of such intermediary conditions should be taken into account.

\section{Conclusions}

Our study suggests that a higher dietary intake of certain (poly)phenols may be potentially associated with better sleep quality. However, further epidemiological studies are needed to confirm the presented hypothesis, with a major focus on sleep quality. Several aspects should be further considered in future studies, such as the use of caffeinated beverages or the timing of food and alcohol intake. Future studies should additionally focus on the inter-individual variation in response to the consumption of (poly)phenols and thus investigate the associations not only for their dietary intake but also for the true internal exposure to their metabolites. In this context, attention to the gut microbiota composition should also be paid as differences in microbial species may condition (poly)phenol metabolite formation and bioactivity. Finally, intervention studies will be needed to explore the level of absorption and bioavailability of dietary (poly)phenols and the characterization of biologically available (poly)phenol metabolites responsible for the promotion of resilience against cognitive impairment in response to poor sleep quality.

Author Contributions: Conceptualization, J.G., R.F., F.C., F.G., G.G.; methodology J.G., R.F., F.C., F.G., G.G.; validation, J.G., R.F., S.C., F.C., F.G., G.G.; formal analysis, J.G. and G.G.; investigation, J.G., R.F., F.C., F.G., G.G.; data curation, J.G. and S.C.; writing-original draft preparation, J.G., R.F., F.C., F.G., G.G.; writing-review and editing, J.G., R.F., S.C., D.A., P.M., D.D.R., F.C., F.G., G.G.; supervision, R.F., F.C., F.G., G.G. All authors have read and agreed to the published version of the manuscript.

Funding: This study was partially supported by a fund from the Italian Ministry of Health "Ricerca Corrente" (RC n. 2751594) (Ferri, Caraci and Godos).

Conflicts of Interest: The authors declare no conflict of interest.

\section{References}

1. Afshin, A.; Sur, P.J.; Fay, K.A.; Cornaby, L.; Ferrara, G.; Salama, J.S.; Mullany, E.C.; Abate, K.H.; Abbafati, C. Health effects of dietary risks in 195 countries, 1990-2017: A systematic analysis for the Global Burden of Disease Study 2017. Lancet 2019, 393, 1958-1972. [CrossRef] 
2. Stanaway, J.D.; Afshin, A.; Gakidou, E.; Lim, S.S.; Abate, D.; Abate, K.H.; Abbafati, C.; Abbasi, N.; Abbastabar, H.; Abd-Allah, F.; et al. Global, regional, and national comparative risk assessment of 84 behavioural, environmental and occupational, and metabolic risks or clusters of risks for 195 countries and territories, 1990-2017: A systematic analysis for the Global Burden of Disease Study 2017. Lancet 2018, 392, 1923-1994. [CrossRef]

3. Angelino, D.; Godos, J.; Ghelfi, F.; Tieri, M.; Titta, L.; Lafranconi, A.; Marventano, S.; Alonzo, E.; Gambera, A.; Sciacca, S.; et al. Fruit and vegetable consumption and health outcomes: An umbrella review of observational studies. Int. J. Food Sci. Nutr. 2019, 70, 652-667. [CrossRef] [PubMed]

4. Godos, J.; Tieri, M.; Ghelfi, F.; Titta, L.; Marventano, S.; Lafranconi, A.; Gambera, A.; Alonzo, E.; Sciacca, S.; Buscemi, S.; et al. Dairy foods and health: An umbrella review of observational studies. Int. J. Food Sci. Nutr. 2019, 71, 138-151. [CrossRef] [PubMed]

5. Grosso, G.; Godos, J.; Galvano, F.; Giovannucci, E.L. Coffee, Caffeine, and Health Outcomes: An Umbrella Review. Annu. Rev. Nutr. 2017, 37, 131-156. [CrossRef]

6. Marventano, S.; Godos, J.; Tieri, M.; Ghelfi, F.; Titta, L.; Lafranconi, A.; Gambera, A.; Alonzo, E.; Sciacca, S.; Buscemi, S.; et al. Egg consumption and human health: An umbrella review of observational studies. Int. J. Food Sci. Nutr. 2019, 71, 325-331. [CrossRef]

7. Tieri, M.; Ghelfi, F.; Vitale, M.; Vetrani, C.; Marventano, S.; Lafranconi, A.; Godos, J.; Titta, L.; Gambera, A.; Alonzo, E.; et al. Whole grain consumption and human health: An umbrella review of observational studies. Int. J. Food Sci. Nutr. 2020, 1-10. [CrossRef]

8. Schwingshackl, L.; Hoffmann, G.; Missbach, B.; Stelmach-Mardas, M.; Boeing, H. An Umbrella Review of Nuts Intake and Risk of Cardiovascualr Disease. Curr. Pharm. Des. 2017, 22, 1. [CrossRef]

9. Huang, Q.; Liu, H.; Suzuki, K.; Ma, S.; Liu, C. Linking What We Eat to Our Mood: A Review of Diet, Dietary Antioxidants, and Depression. Antioxidants 2019, 8, 376. [CrossRef]

10. Dominguez, L.J.; Barbagallo, M.; Garcia, M.M.; Godos, J.; Martinez-Gonzalez, M.A. Dietary Patterns and Cognitive Decline: Key features for prevention. Curr. Pharm. Des. 2019, 25, 2428-2442. [CrossRef]

11. Manna, P.; Jain, S.K. Obesity, Oxidative Stress, Adipose Tissue Dysfunction, and the Associated Health Risks: Causes and Therapeutic Strategies. Metab. Syndr. Relat. Disord. 2015, 13, 423-444. [CrossRef] [PubMed]

12. St-Onge, M.-P.; Mikic, A.; Pietrolungo, C.E. Effects of Diet on Sleep Quality. Adv. Nutr. 2016, 7, 938-949. [CrossRef] [PubMed]

13. Godos, J.; Currenti, W.; Angelino, D.; Mena, P.; Castellano, S.; Caraci, F.; Galvano, F.; Del Rio, D.; Ferri, R.; Grosso, G. Diet and mental health: Review of the recent updates on molecular mechanisms. Antioxidants 2020, 9, 346. [CrossRef]

14. Kanagasabai, T.; Ardern, C.I. Inflammation, Oxidative Stress, and Antioxidants Contribute to Selected Sleep Quality and Cardiometabolic Health Relationships: A Cross-Sectional Study. Mediat. Inflamm. 2015, 2015, 824589. [CrossRef] [PubMed]

15. Kanagasabai, T.; Ardern, C.I. Contribution of Inflammation, Oxidative Stress, and Antioxidants to the Relationship between Sleep Duration and Cardiometabolic Health. Sleep 2015, 38, 1905-1912. [CrossRef] [PubMed]

16. Pace-Schott, E.F.; Spencer, R. Sleep-Dependent Memory Consolidation in Healthy Aging and Mild Cognitive Impairment. Curr. Top. Behav. Neurosci. 2014, 25, 307-330.

17. Del Rio, D.; Rodriguez-Mateos, A.; Spencer, J.P.; Tognolini, M.; Borges, G.; Crozier, A. Dietary (poly)phenolics in human health: Structures, bioavailability, and evidence of protective effects against chronic diseases. Antioxid. Redox Signal. 2012, 18, 1818-1892. [CrossRef]

18. Mena, P.; Domínguez-Perles, R.; Gironés-Vilaplana, A.; Baenas, N.; García-Viguera, C.; Villaño, D. Flavan-3-ols, anthocyanins, and inflammation. IUBMB Life 2014, 66, 745-758. [CrossRef]

19. Rodriguez-Mateos, A.; Vauzour, D.; Krueger, C.G.; Shanmuganayagam, D.; Reed, J.; Calani, L.; Mena, P.; Del Rio, D.; Crozier, A. Bioavailability, bioactivity and impact on health of dietary flavonoids and related compounds: An update. Arch. Toxicol. 2014, 88, 1803-1853. [CrossRef]

20. Fraga, C.G.; Croft, K.D.; Kennedy, D.O.; Tomas-Barberan, F. The effects of polyphenols and other bioactives on human health. Food Funct. 2019, 10, 514-528. [CrossRef]

21. Gomez-Pinilla, F.; Nguyen, T.T.J. Natural mood foods: The actions of polyphenols against psychiatric and cognitive disorders. Nutr. Neurosci. 2012, 15, 127-133. [CrossRef] [PubMed] 
22. Brickman, A.M.; Khan, U.A.; Provenzano, F.A.; Yeung, L.-K.; Suzuki, W.; Schroeter, H.; Wall, M.; Sloan, R.P.; Small, S.A. Enhancing dentate gyrus function with dietary flavanols improves cognition in older adults. Nat. Neurosci. 2014, 17, 1798-1803. [CrossRef] [PubMed]

23. Godos, J.; Ferri, R.; Caraci, F.; Cosentino, F.I.I.; Castellano, S.; Galvano, F.; Grosso, G. Adherence to the Mediterranean Diet is Associated with Better Sleep Quality in Italian Adults. Nutrients 2019, 11, 976. [CrossRef] [PubMed]

24. Godos, J.; Ferri, R.; Caraci, F.; Cosentino, F.I.I.; Castellano, S.; Shivappa, N.; Hébert, J.R.; Galvano, F.; Grosso, G. Dietary Inflammatory Index and Sleep Quality in Southern Italian Adults. Nutrients 2019, 11, 1324. [CrossRef] [PubMed]

25. Grosso, G.; Marventano, S.; D’Urso, M.; Mistretta, A.; Galvano, F. The Mediterranean healthy eating, ageing, and lifestyle (MEAL) study: Rationale and study design. Int. J. Food Sci. Nutr. 2017, 68, 577-586. [CrossRef]

26. Craig, C.L.; Marshall, A.; Sjöström, M.; Bauman, A.E.; Booth, M.L.; Ainsworth, B.E.; Pratt, M.; Ekelund, U.; Yngve, A.; Sallis, J.F.; et al. International Physical Activity Questionnaire: 12-Country Reliability and Validity. Med. Sci. Sports Exerc. 2003, 35, 1381-1395. [CrossRef]

27. Mannocci, A.; Di Thiene, D.; Del Cimmuto, A.; Masala, D.; Boccia, A.; De Vito, E.; La Torre, G. International Physical Activity Questionnaire: Validation and assessment in an Italian sample. Ital. J. Public Health 2010, 7 , 369-376.

28. Mistretta, A.; Marventano, S.; Platania, A.; Godos, J.; Galvano, F.; Grosso, G. Metabolic profile of the Mediterranean healthy Eating, Lifestyle and Aging (MEAL) study cohort. Mediterr. J. Nutr. Metab. 2017, 10, 131-140. [CrossRef]

29. Buscemi, S.; Rosafio, G.; Vasto, S.; Massenti, F.M.; Grosso, G.; Galvano, F.; Rini, N.; Barile, A.M.; Maniaci, V.; Cosentino, L.; et al. Validation of a food frequency questionnaire for use in Italian adults living in Sicily. Int. J. Food Sci. Nutr. 2015, 66, 426-438. [CrossRef]

30. Marventano, S.; Mistretta, A.; Platania, A.; Galvano, F.; Grosso, G. Reliability and relative validity of a food frequency questionnaire for Italian adults living in Sicily, Southern Italy. Int. J. Food Sci. Nutr. 2016, 67, 857-864. [CrossRef]

31. Istituto Nazionale di Ricerca per gli Alimenti e la Nutrizione. Tabelle di Composizione degli Alimenti; CREA: Rome, Italy, 2009.

32. Godos, J.; Marventano, S.; Mistretta, A.; Galvano, F.; Grosso, G. Dietary sources of polyphenols in the Mediterranean healthy Eating, Aging and Lifestyle (MEAL) study cohort. Int. J. Food Sci. Nutr. 2017, 68, 750-756. [CrossRef] [PubMed]

33. Neveu, V.; Pérez-Jiménez, J.; Vos, F.; Crespy, V.; Du Chaffaut, L.; Mennen, L.; Knox, C.; Eisner, R.; Cruz, J.; Wishart, D.; et al. Phenol-Explorer: An online comprehensive database on polyphenol contents in foods. Database 2010, 2010, bap024. [CrossRef] [PubMed]

34. Rothwell, J.A.; Pérez-Jiménez, J.; Neveu, V.; Medina-Remón, A.; M’Hiri, N.; García-Lobato, P.; Manach, C.; Knox, C.; Eisner, R.; Wishart, D.S.; et al. Phenol-Explorer 3.0: A major update of the Phenol-Explorer database to incorporate data on the effects of food processing on polyphenol content. Database 2013, 2013, bat070. [CrossRef]

35. Bognar, A. Tables on Weight Yield of Food and Retention Factors of Food Constituents for the Calculation of Nutrient Composition of Cooked Foods (Dishes); Bundesforschungsanstalt für Ernährung: Karlsruhe, Germany, 2002.

36. Willett, W.C. Reproducibility and Validity of Food-Frequency Questionnaires. In Nutritional Epidemiology; Oxford University Press (OUP): New York, NY, USA, 1998; pp. 101-147.

37. Buysse, D.J.; Reynolds, C.F.; Monk, T.H.; Berman, S.R.; Kupfer, D.J. The Pittsburgh sleep quality index: A new instrument for psychiatric practice and research. Psychiatry Res. Neuroimaging 1989, 28, 193-213. [CrossRef]

38. Curcio, G.; Tempesta, D.; Scarlata, S.; Marzano, C.; Moroni, F.; Rossini, P.M.; Ferrara, M.; De Gennaro, L. Validity of the Italian Version of the Pittsburgh Sleep Quality Index (PSQI). Neurol. Sci. 2012, 34, 511-519. [CrossRef]

39. Noorwali, E.; Hardie, L.J.; Cade, J.E. Fruit and Vegetable Consumption and Their Polyphenol Content Are Inversely Associated with Sleep Duration: Prospective Associations from the UK Women's Cohort Study. Nutrients 2018, 10, 1803. [CrossRef]

40. Noorwali, E.; Cade, J.E.; Burley, V.J.; Hardie, L.J. The relationship between sleep duration and fruit/vegetable intakes in UK adults: A cross-sectional study from the National Diet and Nutrition Survey. BMJ Open 2018, 8, e020810. [CrossRef] 
41. Jansen, E.C.; She, R.; Rukstalis, M.M.; Alexander, G.L. Sleep Duration and Quality in Relation to Fruit and Vegetable Intake of US Young Adults: A Secondary Analysis. Int. J. Behav. Med. 2020, 2020, 1-12. [CrossRef]

42. Zhao, W.; Li, Y.; Ma, W.; Ge, Y.; Huang, Y. A study on quality components and sleep-promoting effects of GABA black tea. Food Funct. 2015, 6, 3393-3398. [CrossRef]

43. Socci, V.; Tempesta, D.; Desideri, G.; De Gennaro, L.; Ferrara, M. Enhancing Human Cognition with Cocoa Flavonoids. Front. Nutr. 2017, 4. [CrossRef]

44. Godos, J.; Castellano, S.; Ray, S.; Grosso, G.; Galvano, F. Dietary Polyphenol Intake and Depression: Results from the Mediterranean Healthy Eating, Lifestyle and Aging (MEAL) Study. Molecules 2018, $23,999$. [CrossRef] [PubMed]

45. Chang, S.-C.; Cassidy, A.; Willett, W.C.; Rimm, E.B.; O’Reilly, E.J.; Okereke, O.I. Dietary flavonoid intake and risk of incident depression in midlife and older women. Am. J. Clin. Nutr. 2016, 104, 704-714. [CrossRef]

46. Mikolajczyk, R.; El Ansari, W.; Maxwell, A.E. Food consumption frequency and perceived stress and depressive symptoms among students in three European countries. Nutr. J. 2009, 8, 31. [CrossRef] [PubMed]

47. Liu, C.; Xie, B.; Chou, C.-P.; Koprowski, C.; Zhou, D.; Palmer, P.; Sun, P.; Guo, Q.; Duan, L.; Sun, X.; et al. Perceived stress, depression and food consumption frequency in the college students of China seven cities. Physiol. Behav. 2007, 92, 748-754. [CrossRef]

48. Laugero, K.; Falcon, L.M.; Tucker, K.L. Relationship between perceived stress and dietary and activity patterns in older adults participating in the Boston Puerto Rican Health Study. Appetite 2010, 56, 194-204. [CrossRef]

49. Errisuriz, V.L.; Pasch, K.E.; Perry, C.L. Perceived stress and dietary choices: The moderating role of stress management. Eat. Behav. 2016, 22, 211-216. [CrossRef]

50. Youdim, K.A.; Qaiser, M.; Begley, D.J.; Rice-Evans, C.A.; Abbott, N.J. Flavonoid permeability across an in situ model of the blood-brain barrier. Free Radic. Boil. Med. 2004, 36, 592-604. [CrossRef]

51. Angelino, D.; Carregosa, D.; Domenech-Coca, C.; Savi, M.; Figueira, I.; Brindani, N.; Jang, S.; Lakshman, S.; Molokin, A.; Urban, J.J.F.; et al. 5-(Hydroxyphenyl)- $\gamma$-Valerolactone-Sulfate, a Key Microbial Metabolite of Flavan-3-ols, Is Able to Reach the Brain: Evidence from Different in Silico, In Vitro and In Vivo Experimental Models. Nutrients 2019, 11, 2678. [CrossRef]

52. Dias, G.P.; Cavegn, N.; Nix, A.; Bevilaqua, M.C.D.N.; Stangl, R.; Zainuddin, M.S.A.; Nardi, A.E.; Gardino, P.F.; Thuret, S. The Role of Dietary Polyphenols on Adult Hippocampal Neurogenesis: Molecular Mechanisms and Behavioural Effects on Depression and Anxiety. Oxidative Med. Cell. Longev. 2012, 2012, 541971. [CrossRef]

53. Moghadam, F.H.; Mesbah-Ardakani, M.; Esfahani, M.H.N. Ferulic Acid exerts concentration-dependent anti-apoptotic and neuronal differentiation-inducing effects in PC12 and mouse neural stem cells. Eur. J. Pharmacol. 2018, 841, 104-112. [CrossRef]

54. Kurauchi, Y.; Hisatsune, A.; Isohama, Y.; Mishima, S.; Katsuki, H. Caffeic acid phenethyl ester protects nigral dopaminergic neurons via dual mechanisms involving haem oxygenase-1 and brain-derived neurotrophic factor. Br. J. Pharmacol. 2012, 166, 1151-1168. [CrossRef]

55. Zhao, W.; Wang, J.; Bi, W.; Ferruzzi, M.G.; Yemul, S.; Freire, D.; Mazzola, P.; Ho, L.; Dubner, L.; Pasinetti, G.M. Novel application of brain-targeting polyphenol compounds in sleep deprivation-induced cognitive dysfunction. Neurochem. Int. 2015, 89, 191-197. [CrossRef]

56. Zanoli, P.; Avallone, R.; Baraldi, M. Behavioral characterisation of the flavonoids apigenin and chrysin. Fitoterapia 2000, 71, S117-S123. [CrossRef]

57. Kim, J.-W.; Kim, C.-S.; Hu, Z.; Han, J.-Y.; Kim, S.K.; Yoo, S.-K.; Yeo, Y.M.; Chong, M.S.; Lee, K.; Hong, J.T.; et al. Enhancement of pentobarbital-induced sleep by apigenin through chloride ion channel activation. Arch. Pharmacal Res. 2012, 35, 367-373. [CrossRef]

58. Fernandez, S.P.; Wasowski, C.; Paladini, A.C.; Marder, M. Synergistic interaction between hesperidin, a natural flavonoid, and diazepam. Eur. J. Pharmacol. 2005, 512, 189-198. [CrossRef]

59. Varin, C.; Rancillac, A.; Geoffroy, H.; Arthaud, S.; Fort, P.; Gallopin, T. Glucose Induces Slow-Wave Sleep by Exciting the Sleep-Promoting Neurons in the Ventrolateral Preoptic Nucleus: A New Link between Sleep and Metabolism. J. Neurosci. 2015, 35, 9900-9911. [CrossRef]

60. Garrido, J.; Gaspar, A.; Garrido, J.; Miri, R.; Tavakkoli, M.; Pourali, S.; Saso, L.; Borges, F.; Firuzi, O. Alkyl esters of hydroxycinnamic acids with improved antioxidant activity and lipophilicity protect PC12 cells against oxidative stress. Biochimie 2012, 94, 961-967. [CrossRef] 
61. Tu, Y.; Cheng, S.; Sun, H.-T.; Ma, T.-Z.; Zhang, S. Ferulic acid potentiates pentobarbital-induced sleep via the serotonergic system. Neurosci. Lett. 2012, 525, 95-99. [CrossRef]

62. Ou, Q.; Zheng, Z.; Zhao, Y.; Lin, W. Impact of quercetin on systemic levels of inflammation: A meta-analysis of randomised controlled human trials. Int. J. Food Sci. Nutr. 2019, 71, 152-163. [CrossRef]

63. Rendeiro, C.; Rhodes, J.S.; Spencer, J.P. The mechanisms of action of flavonoids in the brain: Direct versus indirect effects. Neurochem. Int. 2015, 89, 126-139. [CrossRef]

64. Rehman, S.U.; Ali, T.; Alam, S.I.; Ullah, R.; Zeb, A.; Lee, K.W.; Rutten, B.P.F.; Kim, M.O. Ferulic Acid Rescues LPS-Induced Neurotoxicity via Modulation of the TLR4 Receptor in the Mouse Hippocampus. Mol. Neurobiol. 2018, 56, 2774-2790. [CrossRef] [PubMed]

65. Morroni, F.; Sita, G.; Graziosi, A.; Turrini, E.; Fimognari, C.; Tarozzi, A.; Hrelia, P. Neuroprotective Effect of Caffeic Acid Phenethyl Ester in A Mouse Model of Alzheimer's Disease Involves Nrf2/HO-1 Pathway. Aging Dis. 2018, 9, 605-622. [CrossRef] [PubMed]

66. Jung, H.W.; Mahesh, R.; Lee, J.G.; Lee, S.H.; Kim, Y.S.; Park, Y.-K. Pinoresinol from the fruits of Forsythia koreana inhibits inflammatory responses in LPS-activated microglia. Neurosci. Lett. 2010, 480, 215-220. [CrossRef]

67. Lee, K.; Kim, S.-H.; Jeong, E.; Park, J.; Kim, S.; Kim, Y.; Sung, S. New Secoisolariciresinol Derivatives fromLindera obtusilobaStems and Their Neuroprotective Activities. Planta Med. 2009, 76, 294-297. [CrossRef]

68. Rom, S.; Zuluaga-Ramirez, V.; Reichenbach, N.L.; Erickson, M.A.; Winfield, M.; Gajghate, S.; Christofidou-Solomidou, M.; Jordan-Sciutto, K.L.; Persidsky, Y. Secoisolariciresinol diglucoside is a blood-brain barrier protective and anti-inflammatory agent: Implications for neuroinflammation. J. Neuroinflamm. 2018, 15, 25. [CrossRef]

69. Yamagata, K. Do Coffee Polyphenols Have a Preventive Action on Metabolic Syndrome Associated Endothelial Dysfunctions? An Assessment of the Current Evidence. Antioxidants 2018, 7, 26. [CrossRef]

70. Godos, J.; Vitale, M.; Micek, A.; Ray, S.; Martini, D.; Del Rio, D.; Riccardi, G.; Galvano, F.; Grosso, G. Dietary Polyphenol Intake, Blood Pressure, and Hypertension: A Systematic Review and Meta-Analysis of Observational Studies. Antioxidants 2019, 8, 152. [CrossRef]

71. Behl, M.; Bliwise, D.; Veledar, E.; Cunningham, L.; Vázquez, J.; Brigham, K.; Quyyumi, A. Vascular endothelial function and self-reported sleep. Am. J. Med. Sci. 2014, 347, 425-428. [CrossRef]

72. Cooper, D.C.; Ziegler, M.G.; Milic, M.S.; Ancoli-Israel, S.; Mills, P.J.; Loredo, J.S.; Von Känel, R.; Dimsdale, J.E. Endothelial function and sleep: Associations of flow-mediated dilation with perceived sleep quality and rapid eye movement (REM) sleep. J. Sleep Res. 2013, 23, 84-93. [CrossRef]

73. Thomas, S.J.; Calhoun, D. Sleep, insomnia, and hypertension: Current findings and future directions. J. Am. Soc. Hypertens. 2017, 11, 122-129. [CrossRef]

74. Lajous, M.; Rossignol, E.; Fagherazzi, G.; Perquier, F.; Scalbert, A.; Clavel-Chapelon, F.; Boutron-Ruault, M.-C. Flavonoid intake and incident hypertension in women. Am. J. Clin. Nutr. 2016, 103, 1091-1098. [CrossRef]

75. Grosso, G.; Stepaniak, U.; Micek, A.; Kozela, M.; Stefler, D.; Bobak, M.; Pajak, A. Dietary polyphenol intake and risk of hypertension in the Polish arm of the HAPIEE study. Eur. J. Nutr. 2017, 57, 1535-1544. [CrossRef]

76. Godos, J.; Sinatra, D.; Blanco, I.; Mulè, S.; La Verde, M.; Marranzano, M. Association between Dietary Phenolic Acids and Hypertension in a Mediterranean Cohort. Nutrients 2017, 9, 1069. [CrossRef]

77. Suzuki, A.; Nomura, T.; Jokura, H.; Kitamura, N.; Saiki, A.; Fujii, A. Chlorogenic acid-enriched green coffee bean extract affects arterial stiffness assessed by the cardio-ankle vascular index in healthy men: A pilot study. Int. J. Food Sci. Nutr. 2019, 70, 901-908. [CrossRef]

78. Wang, S.; Sarriá, B.; Mateos, R.; Goya, L.; Bravo-Clemente, L. TNF- $\alpha$-induced oxidative stress and endothelial dysfunction in EA.hy926 cells is prevented by mate and green coffee extracts, 5 -caffeoylquinic acid and its microbial metabolite, dihydrocaffeic acid. Int. J. Food Sci. Nutr. 2018, 70, 267-284. [CrossRef]

79. Gautier-Sauvigné, S.; Colas, D.; Parmantier, P.; Clement, P.; Gharib, A.; Sarda, N.; Cespuglio, R. Nitric oxide and sleep. Sleep Med. Rev. 2005, 9, 101-113. [CrossRef]

80. Salvucci, E. The human-microbiome superorganism and its modulation to restore health. Int. J. Food Sci. Nutr. 2019, 70, 781-795. [CrossRef]

81. Ceppa, F.A.; Mancini, A.; Tuohy, K. Current evidence linking diet to gut microbiota and brain development and function. Int. J. Food Sci. Nutr. 2018, 70, 1-19. [CrossRef] 
82. Mörkl, S.; Wagner-Skacel, J.; Lahousen, T.; Lackner, S.; Holasek, S.J.; Bengesser, S.A.; Painold, A.; Holl, A.K.; Reininghaus, E.Z. The Role of Nutrition and the Gut-Brain Axis in Psychiatry: A Review of the Literature. Neuropsychobiology 2018, 79, 80-88. [CrossRef]

83. Caracciolo, B.; Xu, W.; Collins, S.; Fratiglioni, L. Cognitive decline, dietary factors and gut-brain interactions. Mech. Ageing Dev. 2014, 136, 59-69. [CrossRef]

84. Tomas-Barberan, F.; Selma, M.V.; Espín, J.C. Interactions of gut microbiota with dietary polyphenols and consequences to human health. Curr. Opin. Clin. Nutr. Metab. Care 2016, 19, 471-476. [CrossRef] [PubMed]

85. Митев, K.; Taleski, V. Association between the Gut Microbiota and Obesity. Open Access Maced. J. Med. Sci. 2019, 7, 2050-2056. [CrossRef]

86. Ozdal, T.; Sela, D.A.; Ulrih, N.P.; Boyacioglu, D.; Chen, F.; Capanoglu, E. The Reciprocal Interactions between Polyphenols and Gut Microbiota and Effects on Bioaccessibility. Nutrients 2016, 8, 78. [CrossRef] [PubMed]

87. Crispi, S.; Filosa, S.; Di Meo, F. Polyphenols-gut microbiota interplay and brain neuromodulation. Neural Regen Res. 2018, 13, 2055-2059. [CrossRef] [PubMed]

88. Espín, J.C.; González-Sarrías, A.; Tomas-Barberan, F. The gut microbiota: A key factor in the therapeutic effects of (poly)phenols. Biochem. Pharmacol. 2017, 139, 82-93. [CrossRef]

89. Zhi, C.; Huang, J.; Wang, J.; Cao, H.; Bai, Y.; Guo, J.; Su, Z. Connection between gut microbiome and the development of obesity. Eur. J. Clin. Microbiol. Infect. Dis. 2019, 38, 1987-1998. [CrossRef]

(C) 2020 by the authors. Licensee MDPI, Basel, Switzerland. This article is an open access article distributed under the terms and conditions of the Creative Commons Attribution (CC BY) license (http://creativecommons.org/licenses/by/4.0/). 